\title{
Analysis of Noise in Complex-Valued Binary and Bipolar Sigmoid Compressive Sensing
}

\author{
Isidora Stanković, Student Member, IEEE, Miloš Brajović, Student Member, IEEE, \\ Miloš Daković, Member, IEEE, and Ljubiša Stanković, Fellow, IEEE
}

\begin{abstract}
Binary compressive sensing (CS) is a relatively new idea in the theory of sparse signal reconstruction. Under this framework, the signal is reconstructed based on the sign of the available measurements. This paper analyzes basic onebit CS concepts for the case of complex valued random Gaussian measurement matrices. The reconstruction is compared with the B-bit quantized measurements. The concept of binary CS-based reconstruction is generalized by applying a sigmoid function to the measurements. Noise influence is also considered. The reconstruction is performed using a simple iterative thresholding algorithm.

Keywords - compressive sensing, complex, binary, bipolar, sigmoid, reconstruction, sparse signal processing.
\end{abstract}

\section{INTRODUCTION}

COMPREssive sensing (CS) is an intensively studied field of signal processing, dealing with the reconstruction of sparse signals [1]-[7]. Signals are sparse if they can be represented by a small number of non-zero coefficients in some transformation domain. Within the CS framework, such signals can be reconstructed based on a reduced set of measurements. Based on the CS techniques, signal acquisition, transmission and storage can be performed more efficiently, which is crucial in big data setups. The reduced set of measurements (observations, samples) can be a result of an intentional sampling strategy, physical constraints, or a consequence of a signal corruption. In particular, signal samples can be intentionally omitted if they are too heavily corrupted by a noise. This case occurs often in application of some signal processing denoising techniques, such as the L-statistics [4]. During the last decade, the CS theory has been significantly developed, with various reconstruction methods and algorithms being introduced [8]-[13].

In practice, certain signal classes can be considered as sparse in specific transformation domains, e.g. digital images are sparse in a two-dimensional discrete cosine transform domain. Similarly, signals appearing in

Paper received April 06, 2019; accepted April 21, 2019. Date of publication July 31,2019 . The associate editor coordinating the review of this manuscript and approving it for publication was Prof. Branimir Reljin.

This paper is revised and expanded version of the paper presented at the 26th Telecommunications Forum TELFOR 2018 [25].

Authors are with the Faculty of Electrical Engineering, University of Montenegro, Dzordza Vasingotna bb, 81000 Podgorica, Montenegro, e-mail: \{isidoras,milosb,milos,ljubisa\}@ucg.ac.me.

Corresponding author I. Stanković is also with GIPSA Lab, INP Grenoble, University of Grenoble Alpes, 11 Rue des Mathématiques, 38000 Grenoble, France. telecommunications, radar technology, and biomedicine are commonly sparse in the Fourier transform domain, therefore making possible to apply the CS concepts and perform reconstructions based on the reduced sets of samples [12]. Generally speaking, the CS theory inherently assumes that the measurements are taken accurately. However, high accuracy implies a very large number of bits in signal digital forms, which could be, consequently, extremely demanding and expensive for hardware implementations [14]. Therefore, in practice, measurements have to be quantized, meaning that they are represented, acquired, transferred and stored using a limited number of bits.

One-bit measurements represent the most extreme case of quantization. Each measured value is represented by its sign only. Such quantization approach is very robust, memory efficient and simple for sensor design. This paper deals with complex-valued signals, quantized using signs of real and imaginary parts. One-bit measurements can be treated as sign constraints [14]-[17], as opposed to the values to be matched in the mean squared sense during the reconstruction process. As signs of measurements do not provide amplitude information of the signal, the signal can be recovered up to a constant scalar factor only. In the onebit CS framework, by imposing a unity energy constraint on the reconstructed signal, the ambiguity is resolved and the signal reconstruction can be performed [14].

Quantization to one-bit measurements is particularly suitable for hardware systems. The quantizer for the algorithm presented in this paper takes the form of a comparator, which is an inexpensive and fast hardware device. Moreover, one-bit quantizers do not suffer from dynamic range issues [14]-[17]. In [18], a complex form has been used in the direction-of-arrival estimation with a partial Fourier transform matrix as a measurement matrix. Some recent works investigate various aspects of the onebit CS, particularly the influence of noise, such as: application in noisy wireless networks [19], biomedicine [20], robustness in noisy environments [21], [22], [23]; while general reviews regarding this form of CS can be found in [21] and [24].

In this paper, a general Gaussian distributed measurement complex matrix is assumed. The results with a binary complex algorithm are compared with a digital algorithm using $B$ bits with respect to the number of iterations and accuracy, extending the real case analysis from [15]. This paper extends our previous work [25] with the case when the measurements are corrupted with an 
additive noise. The noise in the measurements is zero-mean Gaussian, with various variances.

Generalization of the binary compressive sensing case by using the bipolar sigmoid and hyperbolic tangent functions of the measurement values, as the reference for the reconstruction, is presented as well. The bipolar sigmoid functions are convenient and commonly used instead of sign functions in the neural network configurations [11]. The sign measurements present a special case of the hyperbolic tangent function.

The paper is organized as follows. In Section II, the traditional compressive sensing is briefly explained. The idea of complex-valued binary compressive sensing and the bipolar sigmoid generalization are shown in Section III. In Section IV, the reconstruction algorithm is presented. Results are shown in Section V.

\section{Traditional Compressive SENSING}

Consider a discrete signal $x(n)$ of length $N$, with $0 \leq n \leq N-1$, while $X(k)$ denotes the corresponding transform domain representation. The signal and the corresponding transform are related as

$$
x(n)=\sum_{k=0}^{N-1} X(k) \psi_{k}(n), \quad X(k)=\sum_{n=0}^{N-1} x(n) \phi_{n}(k),
$$

or in a vector form

$$
\mathbf{x}=\boldsymbol{\Psi} \mathbf{X}, \quad \mathbf{X}=\boldsymbol{\Phi} \mathbf{X} .
$$

The matrices $\boldsymbol{\Phi}$ and $\boldsymbol{\Psi}$ are the direct and the inverse transformation matrices, respectively. We assume that the signal is $K$-sparse in the transformation domain, meaning that it has $K \ll N$ nonzero coefficients in that domain.

Following the CS theory, only $M<N$ samples can be used to reconstruct this signal. The signal with $M$ available observations is denoted by $y(m)=x\left(n_{m}\right), m=1,2, \ldots, M$, written in a vector form as:

$$
\mathbf{y}=\left[x\left(n_{1}\right), x\left(n_{2}\right), \ldots, x\left(n_{M}\right)\right]^{T} .
$$

The general goal of CS is to minimize the sparsity of $\mathbf{X}$ using the available observations $\mathbf{y}$ and under the constraints dictated by the measurements equations $\mathbf{y}=\mathbf{A X}$, where the relation between the measurements $\mathbf{y}$ and the transform domain representation $\mathbf{X}$ is modeled using the measurements matrix. In other words, the CS reconstruction goal is to solve

$$
\min \|\mathbf{X}\|_{0} \text { subject to } \mathbf{y}=\mathbf{A X},
$$

where the rows of the $M \times N$ measurement matrix $\mathbf{A}$ correspond to the positions of the available samples. The measurement matrix consists of i.i.d. standard normal distribution coefficients, so that there is no difference between direct/inverse transform framework for the measurements. The $\ell_{0}$-norm used in (3) counts the number of nonzero coefficients in $\mathbf{X}$. However, this norm is not convex and is very sensitive (not applicable) to the noise. This is the reason why a more robust convex $\ell_{1}$-norm is used to measure the sparsity resulting in the minimization problem

$$
\min \|\mathbf{X}\|_{1} \quad \text { subject to } \mathbf{y}=\mathbf{A X} \text {. }
$$

Since the problem is now convex, the gradient descent algorithms can be used. Under certain conditions the solutions of (3) and (4) are the same.

To solve the CS reconstruction problem, many algorithms have been developed. In this paper, a simple thresholding algorithm, presented in Section IV, is used.

\section{BINARY COMPRESSIVE SENSING}

In the CS theory, the number of measurement bits is not commonly considered. Bit-limiting the measurements could significantly affect the reconstruction performance of standard CS approaches. The measurement quantization is particularly important in the hardware implementation context. One-bit measurements are the most extreme and simplest case, realized with comparator-based hardware devices. The one bit represents the sign of the sample

$$
\mathbf{y}=\operatorname{sign}\{\mathbf{A X}\}
$$

which is, for real-valued signals and transforms,

$$
y(m)= \begin{cases}1, & \text { for } m \text {-th element of } \mathbf{A X}>0 \\ -1, & \text { for } m \text {-th element of } \mathbf{A X} \leq 0 .\end{cases}
$$

Then, the CS reconstruction goal can be reformulated as

$$
\min \|\mathbf{X}\|_{1} \text { subject to } \mathbf{y}=\operatorname{sign}\{\mathbf{A X}\} \text {. }
$$

This way, we try to reconstruct the coefficients $X(k)$ by ignoring the amplitude of the available measurements. Consequently, this method needs more measurements, commonly $M>N$, so that the traditional idea of CS is, in that sense, destructed. However, in the case when the quantization accuracy is more important than the number of measurements, we can afford having a larger number of samples [14]. The requirement for storage is also significantly reduced for sign measurements since the total number of bits is still low.

\section{A. Complex-valued extension}

Further, we assume a complex-valued signal $x(n)$. Then the measurements $\mathbf{y}=\mathbf{A X}$ are also complex. We also assume the measurement matrix A consists of random, complexvalued coefficients, i.i.d, normal, zero-mean distribution of real and imaginary parts, with a unity variance

$$
\psi_{k}\left(n_{m}\right) \sim \mathcal{N}(0,1)+j \mathcal{N}(0,1)
$$

for $k=0,1, \ldots, N-1$ and $m=1,2, \ldots, M$. In that case, the measurements are formed as

$$
\mathbf{y}=\operatorname{sign}\{\mathfrak{R}\{\mathbf{A X}\}\}+j \operatorname{sign}\{\mathfrak{I}\{\mathbf{A X}\}\},
$$

where both real and imaginary parts are one-bit quantized.

\section{B. Bipolar sigmoid measurements}

Since the sign function is not differentiable, it is common to use the hyperbolic tangent function instead of the sign function in the corresponding decision processes (like, for example, in the neural networks [11] or signal compression). The measurements are then presented as

$$
\mathbf{y}=\tanh \{\Re\{\alpha \mathbf{A X X}\}\}+j \tanh \{\mathfrak{I}\{\alpha \mathbf{A X X}\}\} .
$$

For a large value of $\alpha$, the hyperbolic tangent function will behave as a sign function. In the vicinity of zero, it will behave as a linear operator. The hyperbolic tangent function with $\alpha=3$ is shown in Fig. 1 . 


\section{BINARY ITERATIVE THRESHOLDING AlgORITHM}

For the reconstruction, we will adapt a method from the family of greedy algorithms, the Binary Iterative Hard Thresholding (BIHT) algorithm presented in [15], [16] for complex-valued signals. It is an extended version of the Iterative Hard Thresholding (IHT) algorithm [10]. This algorithm is convenient for the one-bit compressive sensing framework.

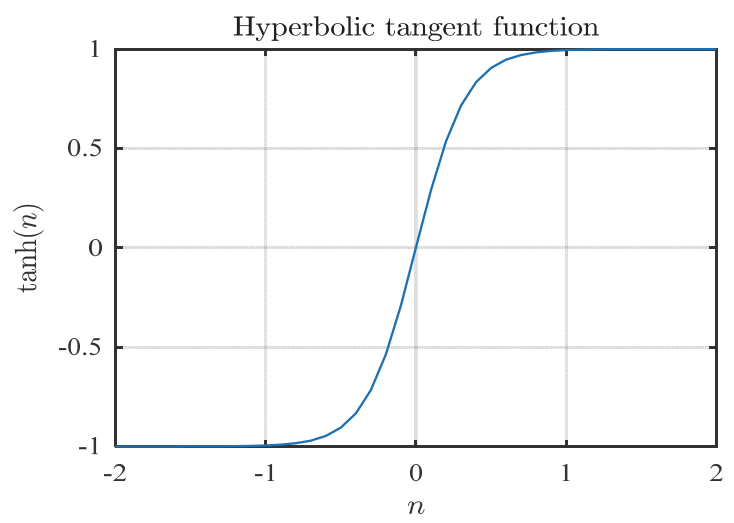

Fig. 1. A hyperbolic tangent function acting as a sigmoid.

For the binary case, the CS based reconstruction is then defined as solving the following problem

$$
\begin{gathered}
\operatorname{argmin}\|\mathbf{y}-\operatorname{sign}\{\mathfrak{R}\{\mathbf{A X}\}\}-j \operatorname{sign}\{\mathfrak{I}\{\mathbf{A X}\}\}\|_{2}^{2} \\
\text { s.t. }\|\mathbf{X}\|_{0}=K .
\end{gathered}
$$

The BIHT algorithm can be divided into three steps.

1. In the first step, the approximation is computed and moved in the gradient direction (in the gradient descent sense). Given that the initial estimate is $\mathbf{X}_{0}=\mathbf{0}$, the value of the signal is updated at the iteration $i$ as

$$
\mathbf{a}_{i+1}=\mathbf{X}_{i}+\frac{\tau}{2} \mathbf{A}^{H}\left[\mathbf{y}-\operatorname{sign}\left\{\mathfrak{R}\left\{\mathbf{A X}_{i}\right\}\right\}-j \operatorname{sign}\left\{\mathfrak{I}\left\{\mathbf{A} \mathbf{X}_{i}\right\}\right\}\right],
$$

where $\tau$ is a constant regulating the gradient descent step.

2. Vector $\mathbf{X}_{i}$ is updated using the largest elements in $\mathbf{a}_{i+1}$

$$
\mathbf{X}_{i+1}=\mathrm{T}_{K}\left\{\mathbf{a}_{i+1}\right\},
$$

where $\mathrm{T}_{K}\{$.$\} is the thresholding operator, taking only the$ $K$ largest components.

The first two steps are repeated until a desired error or the consistency is achieved (or, in the worst case, if the maximum number of iterations is achieved).

3. After the procedure is stopped and the signal is reconstructed, the final step is to normalize the estimate and project the approximation onto the unit $\ell_{2}$ sphere

$$
\mathbf{X}_{R}=\mathbf{X}_{i} /\left\|\mathbf{X}_{i}\right\|_{2} \text {. }
$$

The algorithm is numerically evaluated in Section $\mathrm{V}$.

The stationary case solution of the iterative process $\mathbf{X}_{i+1}=\mathrm{T}_{K}\left\{\mathbf{X}_{i}+\frac{\tau}{2} \mathbf{A}^{H}\left[\mathbf{y}-\mathbf{A} \mathbf{X}_{i}\right]\right\} \quad$ is $\quad \mathbf{X}_{K}=\left(\mathbf{A}_{K}^{H} \mathbf{A}_{K}\right)^{-1} \mathbf{A}_{K}^{H} \mathbf{y}$, where $\mathbf{X}_{K}$ and $\mathbf{A}_{K}$ are obtained by keeping the $K$ nonzero elements of $\mathbf{X}$ and corresponding columns of $\mathbf{A}$.

The binary measurements can be generalized using their
$B$-bit digitalized form. The measurements are stored into Bbit registers,

$$
\mathbf{y}=\operatorname{digital}_{B}\{\mathbf{A X}\}=\lfloor\mathbf{A X}\rfloor_{B} .
$$

In this case, the reconstruction is performed using the iterative matching pursuit algorithm.

The same algorithm can be used for the hyperbolic tangent function, replacing the sign function with tanh.

\section{Results}

Example 1: Consider a signal $x(n)$ of the length $N=256$. The coefficients of the measurement matrix $\mathbf{A}$ are complexvalued i.i.d. normal random variables, that is, $\psi_{k}\left(n_{m}\right) \sim \mathcal{N}(0,1)+j \mathcal{N}(0,1) \quad$ for $\quad k=0,1, \ldots, N-1 \quad$ and $m=1,2, \ldots, M$. The assumed signal is sparse, with sparsity level $K=5$. It is reconstructed using the BIHT algorithm based on $M=3 N$ measurements, with one-bit real and imaginary parts. The gradient-descent step is $\tau=0.001$. The original and the reconstructed signals are compared in Fig. 2.

The reconstruction performance of the BIHT algorithm was also checked in a more extensive numerical experiment. For a signal with sparsity $K=5$, the number of measurements $M$ was varied from 64 to 1024, with step 64 .

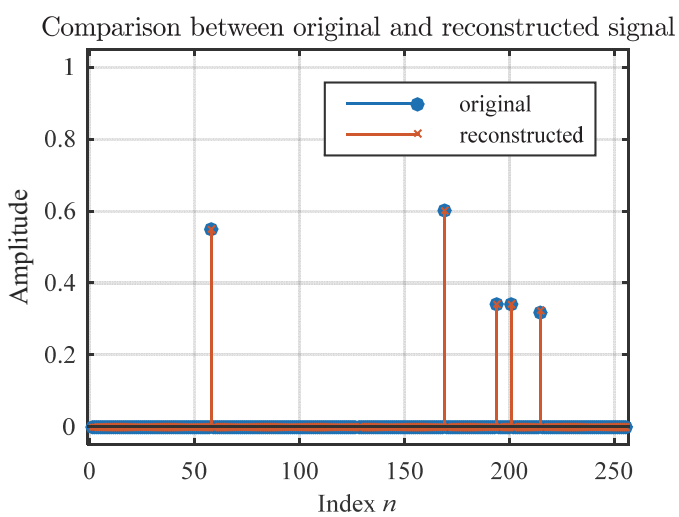

Fig. 2. Reconstruction of a signal using the BIHT algorithm, based on the measurements with one-bit real and imaginary parts. The signal parameters are $N=256, M=3 \times 256$ and $K=5$.

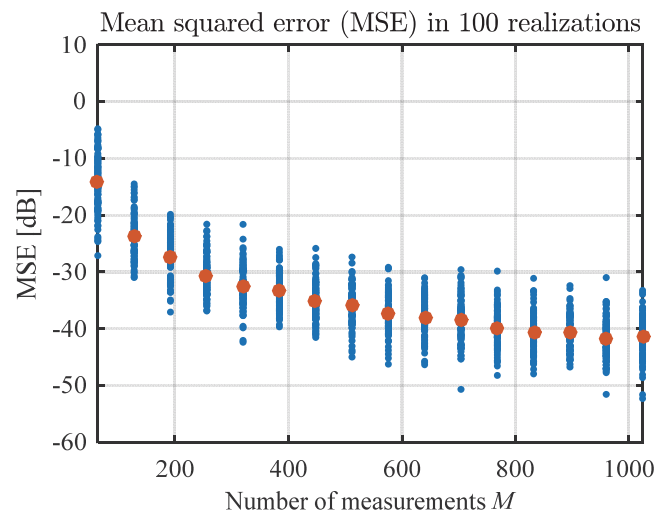

Fig. 3. MSE in the reconstruction of signals with one-bit real and imaginary parts, with length $N=256$ and sparsity $K=5$. The MSE is calculated based on 100 independent realizations of signals and measurements, for each considered number of measurements $M$. Blue dots denote mean squared errors from each realization, red dots represent the MSE obtained by averaging the results from all realizations. 

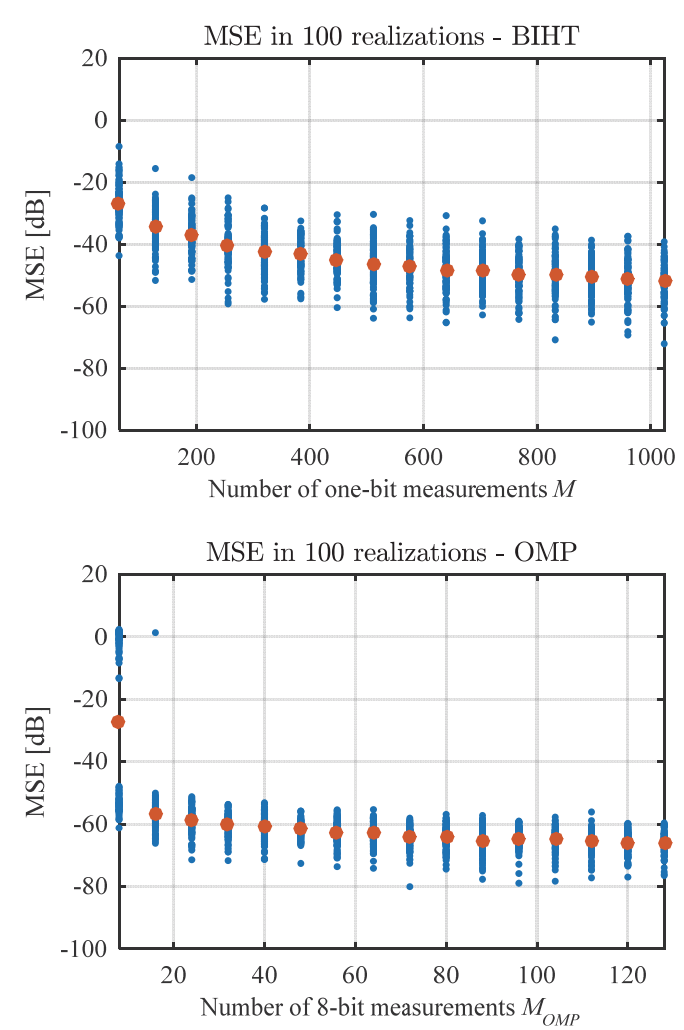

Fig. 4. The mean squared error in the reconstruction of signals with length $N=128$ and sparsity $K=2$ using: the BIHT algorithm (first row) and the OMP algorithm (second row). The MSEs are calculated based on 100 independent realizations of signals for each considered number of measurements. In the OMP signal case, $M_{O M P}$ random 8-bit quantized measurements are considered (real and imaginary parts are quantized independently). In the case of the BIHT, the corresponding number of measurements is $M=8 M_{O M P}$. Blue dots denote mean squared errors in each realization, red dots represent the MSE obtained by averaging the results from all realizations.

For each observed number of measurements $M$, the mean squared error (MSE) is calculated based on 100 independent random realizations of signal with sparsity $K=5$, with corresponding random realizations of matrix $\mathbf{A}$. The results are shown in Fig. 3, where blue dots represent squared errors in each observed realization, whereas red dots show the corresponding MSE values.

Example 2: In this experiment, we observe a signal of length $N=128$, with the sparsity $K=2$. The original, complex-valued measurements $y(m), m=1,2, \ldots, M$ are intentionally brought as inputs of a $B=8$-bit quantizer, to obtain quantized measurements $\tilde{y}(m)$. The real and imaginary parts are quantized independently, each with 8bits. They form $\tilde{y}(m)=\lfloor\mathfrak{R}\{\mathbf{A X}\}\rfloor_{B}+j\lfloor\mathfrak{I}\{\mathbf{A X}\}\rfloor_{B}$, where $\lfloor\cdot\rfloor_{B}$ denotes the $B$-bit quantization. These quantized 8-bit measurements are used as the basis of the CS reconstruction using the classical OMP algorithm from [8], [11], [12]. The number of available 8-bit measurements $M_{O M P}$ was varied from 8 to 128 , with step 8 . The reconstruction MSE was calculated based on 100 independent random realizations of signal with various, randomly positioned non-zero coefficients with random amplitudes, and with corresponding random coefficients in the measurement matrix $\mathbf{A}$, of the form $\psi_{k}\left(n_{m}\right) \sim \mathcal{N}(0,1)+j \mathcal{N}(0,1)$ for
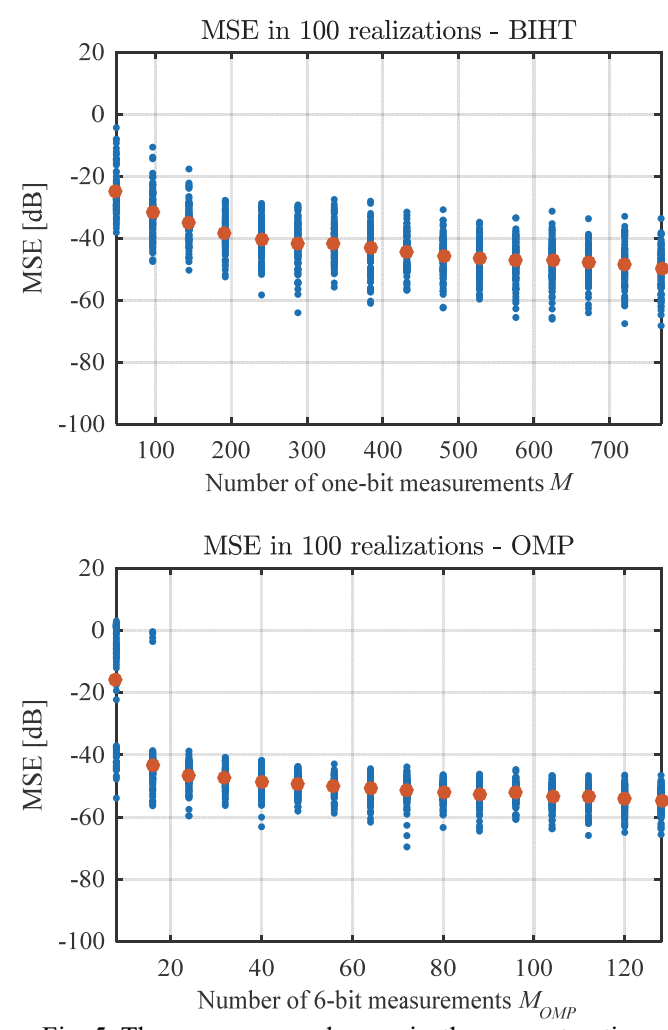

Fig. 5. The mean squared error in the reconstruction of signals with length $N=128$ and sparsity $K=2$ using: the BIHT algorithm (first row) and the OMP algorithm (second row). The MSEs are calculated based on 100 independent realizations of signals for each considered number of measurements. In the OMP signal case, $M_{O M P}$ random 6-bit quantized measurements are considered (real and imaginary parts are quantized independently). In the case of the BIHT, the corresponding number of measurements is $M=6 M_{O M P}$. Blue dots denote mean squared errors in each realization, red dots represent the MSE obtained by averaging the results from all realizations.

$k=0,1, \ldots, N-1 \quad$ and $m=1,2, \ldots, M_{O M P} . \quad$ The obtained results are shown in Fig. 4 (second row), where the quantization with $B=8$ bits increases the MSE level, as compared to the reconstruction using the accurate complexvalued measurements.

Signals with the same properties as in the OMP case are considered in the experiment using the BIHT algorithm with $M=B \cdot M_{O M P}$ one-bit measurements (with real and imaginary one-bit parts).

The reconstruction MSE is shown in Fig. 4 (first row). The MSE slowly reduces as the number of measurements increases, but the BIHT algorithm does not reach the level of the OMP MSE, for the observed number of measurements, and for the observed quantization levels.

To additionally check the influence of the quantization on the OMP reconstruction, we reduce the number of measurement bits to $B=6$, and perform the same experiment. The results are presented in Fig. 5. They indicate that, in this case, the OMP and the BIHT reconstruction performance are similar. With an increase of the number of available one-bit measurements, the MSE of the BIHT reduces proportionally. It is important to note that the main advantage of the BIHT reconstruction is in a much simpler hardware implementation.

Example 3: In this example, we shall consider a signal of length $N=256$, with noisy measurements 


$$
\mathbf{y}=\operatorname{sign}\{\mathfrak{R}\{\mathbf{A X}+\boldsymbol{\varepsilon}\}\}+j \operatorname{sign}\{\mathfrak{I}\{\mathbf{A X}+\boldsymbol{\varepsilon}\}\},
$$

where $\boldsymbol{\varepsilon}$ is a Gaussian noise vector with zero-mean and variance $\sigma^{2}$. The variance is varied from $10^{-3}$ to 1 . The BIHT algorithm is executed with $\tau=0.001$. The MSE and the signal-to-noise ratio (SNR) values are used to evaluate the reconstruction quality.
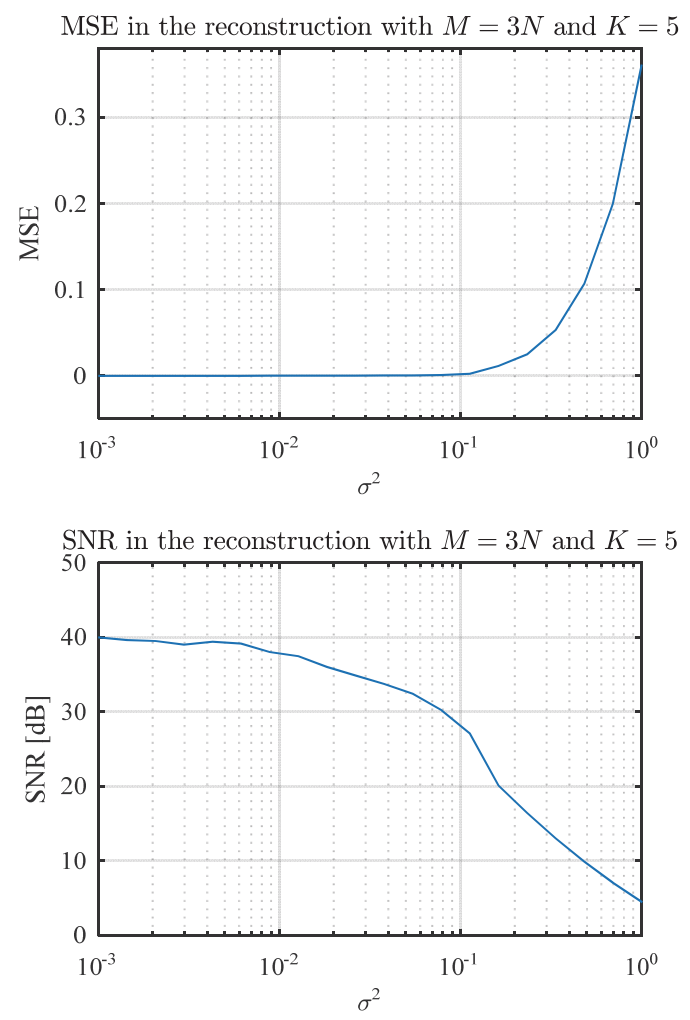

Fig. 6. MSE and SNR of the reconstruction of signals in 100 realizations, with various noise levels and $M=3 N$ and $K=5$.

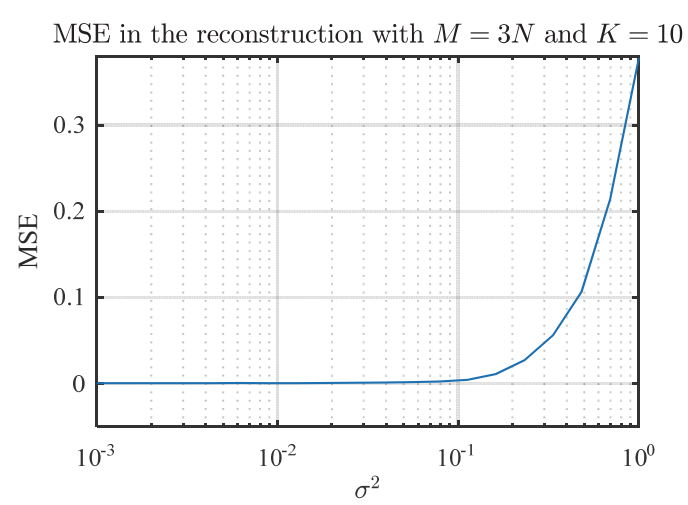

SNR in the reconstruction with $M=3 N$ and $K=10$

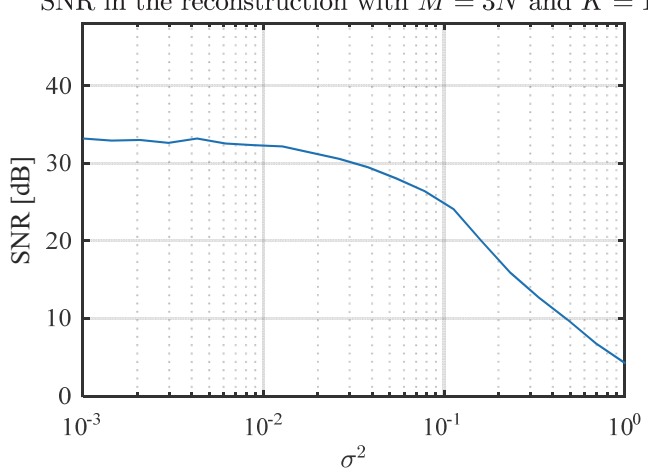

Fig. 7. MSE and SNR of the reconstruction of signals in 100 realizations, with various noise levels and $M=3 N$ and $K=10$.
The error, averaged over 100 independent signal and noise realizations, with $K=5$ and $M=3 N$, is shown in Fig. 6 . The case when the same number of measurements was taken, but with the sparsity $K=10$, is shown in Fig. 7 .
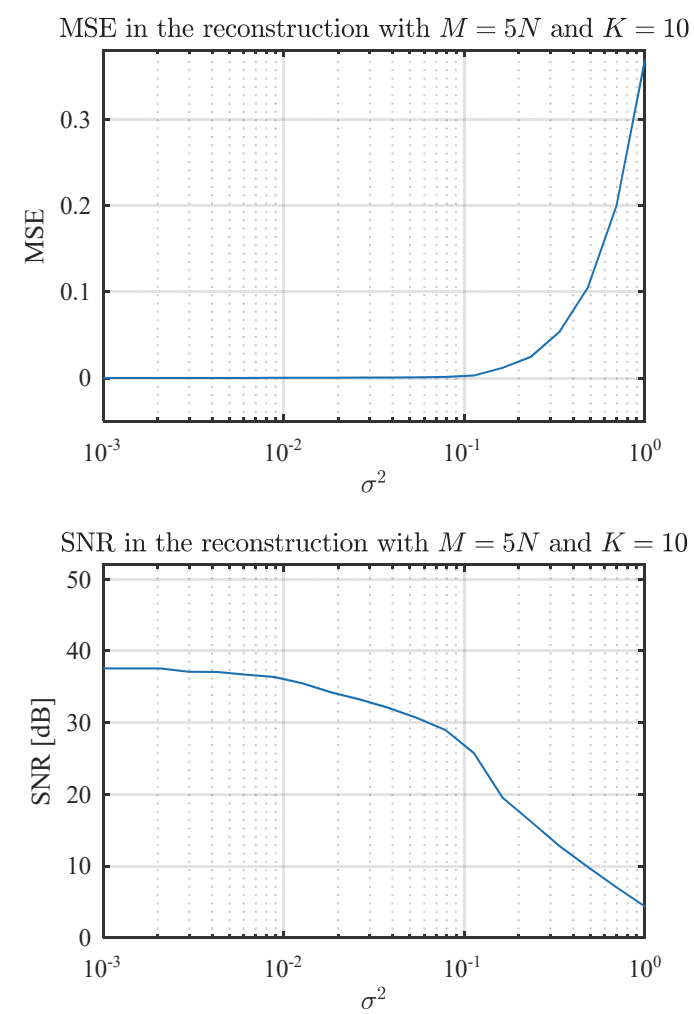

Fig. 8. MSE and SNR of the reconstruction of signals in 100 realizations, with various noise levels and $M=5 N$ and $K=10$.

MSE in the bipolar sigmoid measurements reconstruction

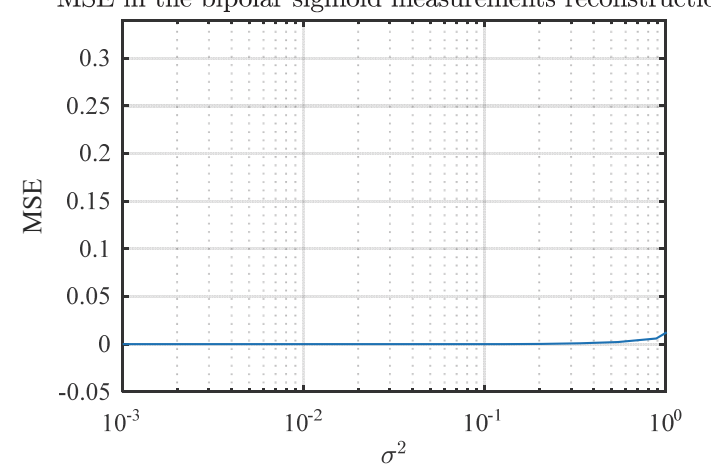

SNR in the bipolar sigmoid measurements reconstruction

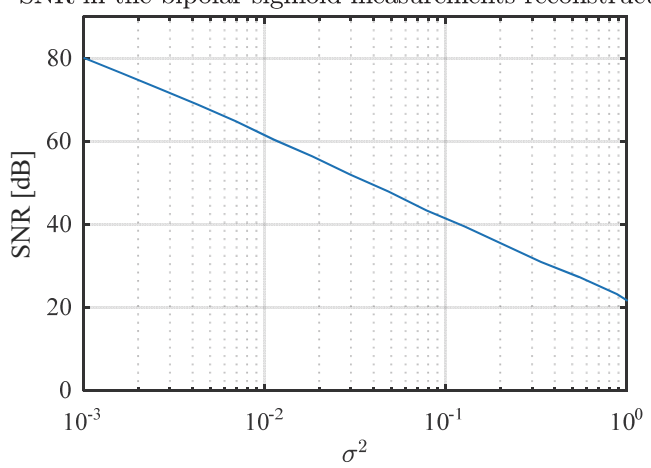

Fig. 9. MSE and SNR of the reconstruction of signals with hyperbolic tangent function measurements in 100 realizations, with various noise levels and $M=3 N$ and $K=5$. 
Finally, the case with an increased number of measurements, when the sparsity level is $K=10$ and $M=$ $5 N$ measurements, is shown in Fig. 8.

In all three cases, acceptable reconstruction results are achieved for a wide range of noise variances. The reconstruction was not successful only for a very large noise level.

Example 4: As mentioned previously, the hyperbolic tangent function can be used instead of the sign function in the corresponding measurement process. In this example, we present the reconstruction of noisy measurements

$$
\mathbf{y}=\tanh \{\mathfrak{R}\{\alpha \mathbf{A X}+\boldsymbol{\varepsilon}\}\}+j \tanh \{\mathfrak{I}\{\alpha \mathbf{A X}+\boldsymbol{\varepsilon}\}\}
$$

with the same signal form as in Example 3 and $\alpha=3$. It is assumed that the signals are sparse with the sparsity $K=5$. The signal is reconstructed using the BIHT algorithm and $M=3 N$ measurements. Step 1 of the BIHT algorithm is adapted to this form of measurements by changing the sign function with tanh function. The MSE and SNR in $\mathrm{dB}$ are presented in Fig. 9. It can be seen that the reconstruction based on hyperbolic tangent measurements produced better results than by using the signs of the measurements.

\section{CONCLUSIONS}

This paper investigates the compressed sensing reconstruction of signals with binary measurements. For the case of complex-valued signals, the binary compressive sensing produced similar reconstruction results as in the case of using the all-real one-bit version of the approach.

We compared the reconstruction results with the classical OMP-based signal recovery from a reduced set of quantized measurements. The measurements quantized to 6 and 8 bits are considered in this context. The traditional CS reconstruction cannot be used on one-bit measurements. The reconstruction results obtained by using the one-bit BIHT are comparable with the standard CS recovery based on the quantized measurements, assuming a similar number of the total measurements bits.

The BIHT showed good reconstruction in both noiseless and noisy cases for the reconstruction of the signals. The generalization of the near-binary measurements was also presented, by using the hyperbolic tangent functions of the measurements as the reference. The presented results indicate that the reconstruction performance is better when bipolar sigmoid is used instead of sign functions for the measurements.

\section{REFERENCES}

[1] D. Donoho, "Compressed sensing," IEEE Transactions on Information Theory, vol. 52, no. 4, 2006, pp. 1289--1306.

[2] Baraniuk CS : R. Baraniuk, "Compressive sensing," IEEE Signal Processing Magazine, vol. 24, no. 4, 2007, pp. 118--121.

[3] E. Candes, J. Romberg and T. Tao. "Robust uncertainty principles: Exact signal reconstruction from highly incomplete frequency information," IEEE Transactions on Information Theory, vol. 52, pp. 489--509, 2006.

[4] L. Stanković, S. Stanković, and M. Amin, "Missing Samples Analysis in Signals for Applications to L-estimation and
Compressive Sensing," Signal Processing, vol. 94, Jan 2014, pp. 401-408

[5] M. Davenport, M. Duarte, Y. Eldar, G. Kutyniok, "Introduction to compressed sensing," Chapter in Compressed Sensing: Theory and Applications, Cambridge University Press, 2012.

[6] L. Stanković, and M. Daković, "On the Uniqueness of the Sparse Signals Reconstruction Based on the Missing Samples Variation Analysis," Mathematical Problems in Engineering, vol. 2015, Article ID 629759, 14 pages, 2015. doi:10.1155/2015/629759

[7] S. Stanković, L. Stanković, and I. Orović, "A Relationship between the Robust Statistics Theory and Sparse Compressive Sensed Signals Reconstruction," IET Signal Processing, vol. 8, no. 3, May 2014.

[8] D. Needell, J. A. Tropp, "CoSaMP: Iterative signal recovery fromincomplete and inaccurate samples," Applied and Computational Harmonic Analysis, vol.26, no.3, 2009, pp.301--321.

[9] T. Blumensath, "Sampling and reconstructing signals from a union of linearsubspaces," IEEE Transactions. on Information Theory. vol.57, no.7, 2011,pp.4660--4671.

[10] T. Bumensath and M. E. Davies, "Iterative hard thresholding for compressed sensing," Appied and Computational Harmonic Analysis., vol. 23, no. 3, pp. 265--274, 2009.

[11] L. Stanković, Digital Signal Processing with Selected Topics. CreateSpace Independent Publishing Platform, An Amazon.com Company, November 4, 2015.

[12] L. Stanković, E. Sejdić, S. Stanković, M. Daković, and I. Orović "A Tutorial on Sparse Signal Reconstruction and its Applications in Signal Processing" Circuits, Systems \& Signal Processing, published online 01. Aug. 2018, DOI 10.1007/s00034-018-0909-2.

[13] L. Stanković, M. Daković, I. Stanković, and S. Vujović, "On the Errors in Randomly Sampled Nonsparse Signals Reconstructed with a Sparsity Assumption," IEEE Geoscience and Remote Sensing Letters, Vol: 14, Issue: 12, Dec. 2017, pp. 2453 - 2456 , DOI: 10.1109/LGRS.2017.2768664

[14] P. Boufounos, R. Baraniuk, "1-bit Compressive Sensing," 42nd Annual Conference on Information Sciences and Systems, Princeton, NJ, USA, 2008.

[15] L. Jacques, J. N. Laska, P. T. Boufounos, and R. G. Baraniuk, "Robust 1-bit compressive sensing via binary stable embeddings for sparse vectors," IEEE Transactions of Information Theory, vol. 59. no. 4, pp. 2082--2102, 2011.

[16] P. North, "One-Bit Compressive Sensing with Partial Support Information," CMC Senior Theses, paper 1194, [Available online: http://scholarship.claremont.edu/cmc_theses/1194, 2015.

[17] P. T. Boufounos, "Greedy sparse signal reconstruction from sign measurements," 2009 Conference Record of the 43rd Asilomar Conference on Signals, Systems and Computers, CA, USA, 2009.

[18] C. Stockle, J. Munir, A. Mezghani and J. A. Nossek, "1-bit direction of arrival estimation based on Compressed Sensing," IEEE 16th International Workshop on Signal Processing Advances in Wireless Communications (SPAWC) 2015, Stockholm, 2015, pp. 246-250

[19] C. Chen, and J. Wu, "Amplitude-Aided 1-Bit Compressive Sensing Over Noisy Wireless Sensor Networks," IEEE Wireless Communication Letters, vol. 4, no. 5, pp. 473 - 476, October 2015.

[20] X. Han, H. Yang, X. Huang, "Review on One-Bit Compressive Sensing and its Biomedical Applications," 23 $3^{\text {rd }}$ International Conference on Digital Signal Processing (IEEE DSP), Shanghai, China, November 2018.

[21] D. Dai, L. Shen, Y. Xu, N. Zhang, "Noisy 1-bit compressive sensing: models and algorithms," Applied and Computational Harmonic Analysis, vol. 40, no. 1, pp. 1 - 32, January 2016.

[22] H. Zayyani, M. Korki, F. Marvasti, "A Distributed 1-bit Compressed Sensing Algorithm Robust to Impulsive Noise," IEEE Communication Letters, vol. 20, no. 6, pp. 1132 - 1135, June 2016.

[23] X. Huang, et. al., "Robust mixed one-bit compressive sensing," Signal Processing, vol. 162, pp. 161 - 168, September 2019.

[24] Z. Li, W. Xu, X. Zhang, and J. Lin, "A survey on one-bit compressed sensing: theory and applications," Frontiers of Computer Science, vol. 12, no. 2, pp. 217 - 230, April 2018.

[25] I. Stanković, M. Brajović, M. Daković, and LJ. Stanković, "Complex-Valued Binary Compressive Sensing," 26th Telecommunications Forum (TELFOR 2018), November 20 - 21, 2018, Belgrade, Serbia. 